ERRATUM

\title{
Antenna-in-package (AiP) in mm-wave band - ERRATUM
}

\author{
MAHMOUD ALHENAWY AND MARTIN SCHNEIDER
}

doi:10.1017/S1759078712000815, published online by Cambridge University Press 23 January 2013

Due to a mistake in the typesetting process, there was an error in the abstract of this article. The value " $9 \mathrm{GHz}$ " was given incorrectly, instead of " $9 \mathrm{dBi}$ ".

The corrected abstract is reproduced below:

We studied the viability of the embedded wafer level ball grid array (eWLB) package environment as an antenna platform for $77 \mathrm{GHz}$ automotive radar sensors and the effects of package fabrication tolerances on the antenna performance. The investigation of different antenna concepts in the eWLB package and their characterization methods are addressed. The design procedures for electrically large, differentially fed loop antennas in a multilayer package structure are introduced. Two different planar antennas are developed and measured in an eWLB package showing promising results such as a gain of $9 \mathrm{dBi}$ and an impedance bandwidth of $8 \mathrm{GHz}$. An acceptable antenna performance is recorded within the tolerance limits. Therefore, the eWLB package is seen as an appropriate platform for $\mathrm{mm}$-wave antennas and as a good candidate for an antenna-in-package (AiP) concept.

We apologise to the authors and the readers for this mistake.

\section{REFERENCE}

[1] Alhenawy, M.; Schneider, M.: Antenna-in-package (AiP) in mm-wave band. Int. J. Microwave and Wireless Tech., 5 (2013), 55-64. doi: 10.1017/S1759078712000815 